\title{
Gepreal The physiological roles of ICAM-1 and ICAM-2 in neutrophil migration into tissues
}

\author{
Ruth Lyck and Gaby Enzmann
}

\begin{abstract}
Purpose of review
Neutrophil extravasation from the blood into tissues is initiated by tethering and rolling of neutrophils on endothelial cells, followed by neutrophil integrin activation and shear resistant arrest, crawling, diapedesis and breaching the endothelial basement membrane harbouring pericytes. Endothelial intercellular cell adhesion molecule (ICAM)-1 and ICAM-2, in conjunction with ICAM-1 on pericytes, critically contribute to each step. In addition, epithelial ICAM-1 is involved in neutrophil migration to peri-epithelial sites. The most recent findings on the role of ICAM-1 and ICAM-2 for neutrophil migration into tissues will be reviewed here.

\section{Recent findings}

Signalling via endothelial ICAM-1 and ICAM-2 contributes to stiffness of the endothelial cells at sites of chronic inflammation and junctional maturation, respectively. Endothelial ICAM-2 contributes to neutrophil crawling and initiation of paracellular diapedesis, which then proceeds independent of ICAM-2.

Substantial transcellular neutrophil diapedesis across the blood-brain barrier is strictly dependent on endothelial ICAM-1 and ICAM-2. Endothelial ICAM-1 or ICAM-2 is involved in neutrophil-mediated plasma leakage. ICAM-1 on pericytes assists the final step of neutrophil extravasation. Epithelial ICAM-1 rather indirectly promotes neutrophil migration to peri-epithelial sites.
\end{abstract}

\section{Summary}

ICAM-1 and ICAM-2 are involved in each step of neutrophil extravasation, and have redundant but also distinct functions. Analysis of the role of endothelial ICAM-1 requires simultaneous consideration of ICAM-2.

\section{Keywords}

endothelium, extravasation, ICAM-1, ICAM-2, neutrophil

\section{INTRODUCTION}

Neutrophils are short-lived immune cells continuously replenished from haematopoietic stem cells in the bone marrow and represent the first line of defence during acute inflammation. Under healthy conditions, the vast majority of mature neutrophils is retained in the bone marrow and another fraction is sequestrated in the liver, spleen and lung, with only $1-2 \%$ of neutrophils circulating in the blood [1]. In contrast, under inflammatory conditions, high numbers of neutrophils are released into the circulation, allowing them to patrol the body and engage with the activated endothelium at sites of microbial infection or sterile tissue damage. Extravasation of neutrophils occurs at the level of postcapillary venules composed of a layer of endothelial cells, connected by tight and adherens junctions, and the endothelial basement membrane, harbouring pericytes. After extravasation, neutrophils display a variety of microbial defence mechanisms through the release of neutrophil extracellular traps (NETosis), phagocytosis and degranulation. Degranulation of intracellular vesicles can be into the phagosomes or into the extracellular space and is of various natures, for example, cytokines and chemokines, myeloperoxidase, gelatinase or matrix metalloproteinases (reviewed in [2]). Under an acute thread fast trafficking is of outstanding importance, but must be well controlled due to the highly destructive capacity of the neutrophils' defence machinery. Intercellular cell adhesion molecule (ICAM)-1 and ICAM-2 on the endothelial surface in conjunction with ICAM-1 on pericytes and epithelial cells are pivotal for neutrophil trafficking

Theodor Kocher Institute, University of Bern, Bern, Switzerland

Correspondence to Ruth Lyck, Theodor Kocher Institute, University of Bern, Bern, Switzerland. E-mail: ruth.lyck@tki.unibe.ch

Curr Opin Hematol 2015, 22:53-59

DOI: $10.1097 / \mathrm{MOH} .0000000000000103$ 


\section{KEY POINTS}

- Extravasation of neutrophils from the blood circulation into inflamed tissues is a multistep process.

- Endothelial ICAM-1 and ICAM-2, neutrophil ICAM-2, pericyte ICAM-1 and epithelial ICAM-1 play important roles at distinct steps of neutrophil migration into tissues.

- Endothelial ICAM-1 and ICAM-2 have overlapping and also distinct roles in neutrophil extravasation.

- Endothelial ICAM-2 is required for the initiation of paracellular diapedesis.

- Transcellular diapedesis of neutrophils strictly depends on ICAM-1 and ICAM-2.

into tissues, and new findings will be the focus of this brief review.

\section{ICAM-1 AND ICAM-2}

ICAM-1 (CD54) and ICAM-2 (CD102) are transmembrane glycoproteins of the immunoglobulin superfamily. The full-length isoform of ICAM- 1 is composed of five immunoglobulin domains and can be present in various splice isoforms (reviewed in [3"]), whereas ICAM-2 harbours two immunoglobulin domains which are homologues to the first and second immunoglobulin domain of ICAM-1 without any known splice variants [4]. ICAM-1 and ICAM-2 are expressed on the surface of the endothelial cells, platelets, neutrophils $[5,6]$ and various other leukocyte subsets $\left[3^{*}, 7\right]$, whereas fibroblasts, epithelial cells, pericytes and various other cell types solely express ICAM-1 [3"]. The constitutive low level of endothelial ICAM-1 is up-regulated upon stimulation of endothelial pattern-recognition receptors, including Toll-like receptors or cytokine receptors such as tumour necrosis factor (TNF) receptor-1 [8], whereas endothelial ICAM-2 is constitutively expressed at rather high levels [7] and localizes differently to ICAM-1 not only to endothelial surfaces but also to the endothelial cell junctions [9"'].

Information on the contribution of ICAM-1 and ICAM-2 to neutrophil trafficking in vivo is controversial due to the presence of alternate ICAM-1 splice variants in ICAM-1 knockout mice. Three different ICAM-1 knockout mice have been generated

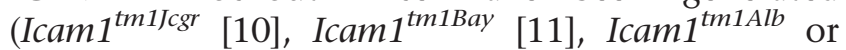
ICAM1 $\left.1^{\text {null }}[12]\right)$, with Icam1 $1^{\text {tm1 Alb }}$ being the only knockout mouse line devoid of any ICAM-1 splice variant (reviewed in [3"]). For ICAM-2, one knockout mouse was created (Icam2 $2^{\text {tm1jcgr }}$ [13]). In a lipopolysaccharide (LPS)-induced endotoxin shock model, Icam $1^{\text {tm1 } 1 \text { cgr }}$ mice had little to no neutrophil infiltration into the liver and were resistant to a lethal dose of LPS, whereas Icam1 $1^{\text {tm1Bay }}$ mice responded with a high mortality and significant neutrophil infiltration into the liver. Here, the discrepant findings on the role of ICAM-1 for leukocyte trafficking were presumably caused by the ICAM- 1 splice isoforms present in the mouse lines employed. The double knockout Icam $1^{\text {null }} /$ Icam $2^{\text {tm1 Icgr }}$ mouse line [14"'], devoid of any ICAM-1 splice isoform, will provide a valuable tool for leukocyte trafficking studies in the future.

\section{ICAM-1 AND ICAM-2 ENGAGE $\beta 2-$ INTEGRINS ON NEUTROPHILS}

ICAM-1 is recognized to bind the $\beta 2$-integrin lymphocyte function-associated antigen (LFA)-1 $(\alpha \mathrm{L} \beta 2, \mathrm{CD} 11 \mathrm{a} / \mathrm{CD} 18)[15,16]$ via its first immunoglobulin domain, whereas macrophage-1 antigen (Mac)-1 ( $\alpha \mathrm{M} \beta 2, \mathrm{CD} 11 \mathrm{~b} / \mathrm{CD} 18)$ [17] engages the third immunoglobulin domain of ICAM-1. LFA-1 is a valid interaction partner of ICAM-2 by binding to its first immunoglobulin domain (reviewed in $\left[3^{\text {"] }}\right)$, whereas only one study shows a direct binding of Mac-1 to ICAM-2 [17]. Upon stimulation, neutrophils can further up-regulate Mac-1 on the cell surface via translocation of Mac-1-containing granules [18]. LFA-1 and Mac-1 adopt changing conformations with different binding strengths to ICAM-1 and ICAM-2: a bend structure of the integrins represents a low-affinity state, an elongated opened conformation of the integrin heterodimer corresponds to an intermediate-affinity state and the elongated closed conformation defines a highaffinity state (reviewed in $[19,20]$ ). Apart from ICAM-1 and ICAM-2, neutrophil LFA-1 and Mac-1 possess further ligands on the endothelial surface such as the receptor for advanced glycation end products (RAGE) [21] or junctional adhesion molecule (JAM)-A and JAM-C (reviewed in [22]).

\section{NEUTROPHIL EXTRAVASATION ACROSS THE VASCULAR ENDOTHELIUM}

Extravasation of neutrophils from the circulation across inflamed post-capillary venules is a multistep process initiated by tethering and rolling of the neutrophil, followed by slow rolling, activation of integrins, shear resistant arrest, crawling, diapedesis and, finally, migration across the pericyte layer (reviewed in $\left[23^{\prime \prime}, 24^{\prime \prime}\right]$ ). A first transient contact between the neutrophil circulating at high velocity in the bloodstream and the inflamed vascular endothelium is mediated through selectins on the 
endothelial surface, namely endothelial (E)-selectin (CD62E) and platelet (P)-selectin (CD62P), and P-selectin glycoprotein ligand (PSGL)-1 on the neutrophil (reviewed in [25]). The high on-off rate and catch-bond properties of the selectin/selectinligand binding $[26,27]$ and neutrophil ICAM-2 interacting in trans with neutrophil LFA-1 to stabilize 'slings' [5] are prominent qualities of neutrophil tethering and rolling, and finally allow the neutrophil to slow down in the presence of high shear $\left[28^{\prime}\right]$. The initial rolling is extremely important for successful neutrophil extravasation as exemplified by severe neutrophilia and recurrent live threatening infections in leukocyte adhesion deficiency (LAD)-II patients lacking the essential selectinligand determinant sialyl Lewis $\mathrm{x}\left(\mathrm{sLe}^{\mathrm{X}}\right)$ on leukocytes (reviewed in [24"]). During rolling, the neutrophil encounters the endothelial cellpresented chemokines or other chemo-attractants that initiate signalling events culminating in the affinity maturation of neutrophil $\beta 2$-integrins, a step dependent on the intracellular integrin adaptor proteins talin-1 and kindlin-3 [29"] (reviewed in [30]). At this point, neutrophils can perform shear resistant arrest via the binding of high-affinity LFA-1 to endothelial ICAM-1 (reviewed in [31]). LAD-I patients who completely lack or only express nonfunctioning $\beta 2$-integrins and LAD-III patients who carry a kindlin-3 mutation preventing proper activation of LFA-1 and Mac-1 suffer from recurrent infections due to impaired neutrophil trafficking to sites of infections and thus emphasize the central role of $\beta 2$-integrins and their ligands ICAM- 1 and ICAM-2 for neutrophil extravasation [24"]. Arrested neutrophils acquire a polarized cell shape with a leading edge in the front and a uropod at the rear end, and then crawl Mac-1-dependent along the luminal surface of the endothelial cells until they reach a site permissive for diapedesis (reviewed in [31]).

Diapedesis of neutrophils occurs predominantly via the paracellular route through the endothelial junctions with minor involvement of the transcellular pathway (reviewed in $[31,32]$ ). The important role of the paracellular diapedesis for neutrophil extravasation was elegantly addressed in vivo by creating a mouse line carrying a knock-in replacement of vascular endothelial (VE)-cadherin - the master regulator of endothelial cell junctions (reviewed in [33"]) - to VE-cadherin- $\alpha$-catenin. This covalent link of $\alpha$-catenin to VE-cadherin rendered the endothelium unresponsive to permeability increasing stimuli, as demonstrated in the skin upon local injection of VE growth factor or histamine [34], and significantly reduced neutrophil extravasation into the IL-1 $\beta$-treated cremaster muscle or the lung after systemic LPS challenge. Paracellular diapedesis of neutrophils is envisaged to occur in a zipper-like fashion and involves endothelial cell junctional molecules, as platelet endothelial cell adhesion molecule (PECAM-1), members of the JAM family, ICAM-2, CD99, CD99L2 and endothelial cell-selective adhesion molecule (ESAM)-1 [31,35], which are recycled from the abluminal to the luminal side of the endothelial cell via the lateral border recycling compartment (LBRC) [35]. Transcellular diapedesis of neutrophils requires the formation of a pore through the endothelial cells and therefore involves dynamic morphological changes of the endothelial cell membrane (reviewed in [36]). An increased level of endothelial ICAM-1 has been demonstrated to promote neutrophil diapedesis via the transcellular pathway across TNF- $\alpha$-stimulated human umbilical vein endothelial cells (HUVECs) [37], presumably through its association with caveolae and F-actinrich membrane domains and LBRC containing endothelial cell junctional molecules [35].

\section{Endothelial ICAM-1 induces increased endothelial cell stiffness}

Engagement of endothelial ICAM-1 induces signalling events in the endothelial cells which promote neutrophil extravasation via recruitment of various actin-cytoskeleton adaptor proteins, among which are $\alpha$-Actinin-4 [38], FilaminB [39] and Cortactin $[40,41]$, to the cytoplasmic domain of ICAM-1 (reviewed in [42"]). Schaefer et al. [43"] analysed the differential effects of $\alpha$-Actinin-4, FilaminB and Cortactin on neutrophil extravasation. Upon ICAM- 1 clustering, $\alpha$-Actinin- 4 was the first protein to be recruited to ICAM-1, followed by Cortactin and FilaminB, as shown in vitro upon ICAM-1 antibody engagement on TNF- $\alpha$-stimulated HUVECs transfected with the respective green fluorescent protein-tagged adapter proteins. Employing atomic force microscopy, the authors demonstrated that knockdown of $\alpha$-Actinin- 4 had the strongest impact on the endothelial cell stiffness. Thus, this suggests that $\alpha$-Actinin-4 supports neutrophil crawling by assisting ICAM-1 to withstand traction forces exerted from the dynamic adhesive contacts through neutrophil $\beta 2$-integrins. Atherosclerotic lesions are characterized by increased stiffness and local inflammation with increased leukocyte infiltrates [44]. Complementing the in-vitro findings, Schaefer et al. [43"] reported significantly increased $\alpha$-Actinin-4 levels in vivo in the aortic endothelial cells of the Apolipoprotein E knockout mice, a mouse model for atherosclerosis, and in human aortic atherosclerotic plaque specimen. In summary, $\alpha$-Actinin- 4 appears to be a reliable marker 
for increased stiffness of vascular elements. However, the potential of targeting $\alpha$-Actinin- 4 to reduce leukocyte infiltration at sites of chronic inflammation requires further investigation.

\section{Endothelial ICAM-2 regulates maturation of endothelial junctions}

Similar to endothelial ICAM-1, the cytoplasmic domain of endothelial ICAM-2 is connected to the actin cytoskeleton through binding of $\alpha$-Actinin [45] and members of the ezrin/radixin/moesin (ERM) complex [46,47]. Amsellem et al. [48"] addressed the role of endothelial ICAM-2 in endothelial junction maturation and barrier formation. Knockdown of ICAM-2 in HUVECs led to increased numbers of gaps between the endothelial cells, although the protein level of VE-cadherin remained unchanged. However, when investigating immortalized murine cardiac endotheliomas, the authors observed a lack of VE-cadherin expression. In these endothelial cells, ICAM-2 regulated N-cadherin junctional localization and barrier formation via ERM recruitment and Rac- 1 activation. In vivo, the authors demonstrated that thrombin-induced vascular permeability across the cremaster vasculature was increased in ICAM-2 knockout mice compared to the wild-type controls. In summary, this study elucidates a link between ICAM-2 downstream signalling and regulation of endothelial junctions. The proposed role of ICAM-2-mediated junctional maturation on neutrophil extravasation awaits further confirmation.

\section{Roles of endothelial ICAM-1 and ICAM-2 for neutrophil crawling and diapedesis}

Luminal crawling of neutrophils has been described to depend on neutrophil Mac-1 binding to endothelial ICAM-1 without any role of ICAM-2 or LFA-1, as demonstrated in macrophage inflammatory protein (MIP)-2 or TNF- $\alpha$-superfused cremaster venules [49] or formyl-methionyl-leucyl-phenylalanine (fMLP)-stimulated neutrophils crawling on recombinant ICAM-1 [50]. Halai et al. [9"'] and Gorina et al. [14"'] investigated the role of endothelial ICAM-1 and ICAM-2 for neutrophil crawling and diapedesis across IL-1 $\beta$-stimulated cremaster muscle post-capillary venules and LPS-stimulated blood-brain barrier (BBB) endothelial cells, respectively. Halai et al. [9"'] defined a significant contribution of endothelial ICAM-2 in Mac-1-dependent neutrophil crawling as shown with confocal intravital microscopy (IVM) in the cremaster muscle of wild-type mice, as compared to the ICAM-2 knockout mice bred on a heterozygous LysM-EGFP background to identify neutrophils. As a new finding, the authors demonstrated that ICAM- 2 is only required for the initiation of but not the process of paracellular diapedesis. In-vitro crawling of neutrophils on recombinant ICAM-1 or ICAM-2 was significantly impaired when Mac-1 was blocked by antibody application. Taken together, this study provides important proof for the significant contribution of ICAM-2 to neutrophil crawling and initiation of diapedesis, whereas paracellular diapedesis itself is independent of endothelial ICAM-2.

The endothelial cells of the blood vessels in the central nervous system forming the BBB are highly specialized and represent an exceptional tight endothelial layer through its complex tight junctions (reviewed in [51]). Gorina et al. [14"'] imaged the dynamic interaction of neutrophils with the BBB endothelial cells in vitro under physiological flow using LPS-stimulated primary mouse brain microvascular endothelial cells (pMBMECs), which represent an established in-vitro BBB model $[52,53]$. To address the individual roles of ICAM-1, ICAM-2, Mac-1 and LFA-1, pMBMECs were isolated from Icam $1^{\text {null }}, \operatorname{Icam} 2^{\text {tm1 1/cgr }}, \operatorname{Icam} 1^{\text {null }} / \operatorname{Icam} 2^{\text {tm1Jcgr }}$ or wild-type mice, and neutrophils were isolated from mice deficient for CD11a, CD11b or CD18 or from wild-type mice. Of note, this is the first study of a double knockout Icam $1^{\text {null }} /$ Icam $2^{\text {tm1Jcgr }}$ mouse line devoid of any ICAM-1 splice isoform [14"']. Live cell imaging of the dynamic post-arrest behaviour of the neutrophils revealed a significant contribution of either molecule to neutrophil crawling on the acutely inflamed BBB. Further, these imaging experiments revealed that crawling of wild-type neutrophils on LPS-stimulated wild-type pMBMECs was with the direction of flow. This differs slightly from neutrophil crawling on MIP-2-stimulated cremaster muscle venules where cells moved perpendicular to the direction of flow [54]. This discrepancy might be indicative of variable contributions of endothelial ICAM-1 and ICAM-2 or neutrophil LFA-1 and Mac-1 for crawling, a suggestion supported by the finding that neutrophil crawling on recombinant ICAM-1 alone overlaid with fMLP is with the direction of flow [55]. Finally, crawling of neutrophils on LPSstimulated pMBMECs was a prerequisite for transcellular diapedesis, which occurred at the exceptional rate of about $25 \%$ of all diapedesis events and was strictly dependent on endothelial ICAM-1 and ICAM-2. Thus, at the acutely inflamed BBB, endothelial ICAM-1 and ICAM-2 fulfill essential roles for neutrophil crawling and transcellular diapedesis.

\section{Endothelial ICAM-1 or ICAM-2 is involved in neutrophil-induced vascular permeability}

It is well known that during acute inflammation, neutrophils contribute to increased vascular 
permeability when stimulated with chemoattractants such as C5a, leukotriene $\mathrm{B}_{4}\left(\mathrm{LTB}_{4}\right)$ or fMLP $[56,57]$. In a recent publication, Finsterbusch et al. [58'] investigated the link between the stimulation of neutrophils and increased permeability. The authors delineated that the binding of the neutrophils to endothelial ICAM-1 and ICAM-2 is a prerequisite for the release of TNF- $\alpha$ by neutrophils, which in turn mediates increased vascular permeability. Confocal IVM in cremaster microvessels demonstrated the release of intracellular stores of TNF- $\alpha$ shortly before, during and after diapedesis by neutrophils stimulated with $\mathrm{LTB}_{4}$. TNF- $\alpha$ release was followed by increased plasma protein leakage and a change in pericyte morphology in wild-type but not in TNF receptor-deficient mice. Similarly, chimeric mice with TNF- $\alpha$-deficient neutrophils failed to induce plasma protein leakage in response to chemoattractants. In vitro, only neutrophils adherent to ICAM-1 or ICAM-2 rapidly released TNF- $\alpha$ in response to $\mathrm{C} 5 \mathrm{a}$ or CXCL1, whereas TNF- $\alpha$ release in response to $\mathrm{LTB}_{4}$ was independent of $\beta 2$-integrin adhesion. In vivo, in spite of the neutrophil-induced plasma leakage, the number of extravasated neutrophils at $4 \mathrm{~h}$ after treatment was not increased compared to TNF receptor-deficient mice. However, this does not exclude facilitation of neutrophil extravasation at later time points.

\section{ICAM-1 on the pericytes guides neutrophils across the pericyte layer}

After diapedesis across the endothelial layer, neutrophils have to cross the endothelial basement membrane with its pericytes. Pericytes are found embedded in the endothelial basement membrane of smaller-calibre vessels as pre-capillary arterioles, capillaries and post-capillary venules, and can vary in their phenotype. Whereas most pericytes express smooth muscle $\alpha$-actin and up-regulate ICAM-1 upon inflammatory conditions, only a subgroup is positive for NG2 and shows constitutive ICAM-1 expression $\left[59,60^{-}\right]$. ICAM-1 on pericytes has been demonstrated to support Mac-1-dependent neutrophil crawling in vitro on IL-1 $\beta$-stimulated Thy $1^{+}$ NG $2^{+}$pericytes from placental microvessels [61"] and to facilitate the migration of the neutrophils through gaps in the pericyte layer in vivo in inflamed cremaster venules [62]. This guidance might be explained through the finding that $\mathrm{NG}^{+}{ }^{+}$, but not $\mathrm{NG}^{-}$, pericytes attract neutrophils via secretion of macrophage inhibitory factor (MIF) [60"]. Interestingly, diapedesis of neutrophils through IL-1 $\beta$ stimulated HUVECs in vitro significantly increased both the level of CD18 on the neutrophils and the rate of neutrophil migration across the pericyte layer via ICAM-1 [61"]. Taken together, these recent findings allow suggesting that migration of neutrophils across an endothelial monolayer activates the neutrophils to release TNF- $\alpha$ and up-regulate $\beta 2$-integrin, which in turn increases the ICAM-1 level on pericytes and facilitates migration across the pericyte layer.

\section{ROLE OF ICAM-1 IN THE MIGRATION OF NEUTROPHILS TO PERI-EPITHELIAL SITES}

In inflammatory processes of the intestine, the lung and the brain neutrophils encounter epithelial barriers, namely the intestinal epithelium, the alveolar epithelium and the choroid plexus. So far, neutrophil trans-epithelial migration is considered to be regulated by interaction of the neutrophil Mac-1 with the basolateral aspects of the epithelium [63], and further involves interaction of epithelial CD47 with the neutrophil signal regulatory protein (SIRP)- $\alpha[64,65]$ in some epithelia [66]. Although ICAM-1 is up-regulated in colonic specimen of ulcerative colitis and Crohn's disease patients [67] and is expressed by choroid plexus epithelial cells in the brain $[68,69]$, it is exclusively confined to the apical side of the epithelium and therefore not available for receptor/ligand-mediated neutrophil migration from the basolateral to the luminal compartment.

Sumagin et al. [70"] addressed the role of ICAM-1 on the luminal face of the intestinal epithelial cells for neutrophil migration in a murine intestinal loop model in vivo and on a human interferon (IFN)- $\gamma$ treated intestinal epithelial cell line in vitro. There, ICAM-1 ligation via antibodies or neutrophils on the epithelial cells induced myosin light chain kinase-mediated increased monolayer permeability and enhanced neutrophil basolateral-to-apical diapedesis towards an fMLP gradient in a two-chamberbased experimental set-up. Upon completion of diapedesis, neutrophils showed higher levels of Mac-1, remained adherent to the apical face of the epithelial monolayer and exhibited increased lateral motility. In vivo, intraperitoneal administration of IFN- $\gamma$ and TNF- $\alpha$ increased the ICAM-1 level on the apical face of intestinal epithelial cells. Cross-linking of ICAM-1 with an anti-ICAM-1 antibody injected into the lumen of intestinal loops increased the trans-epithelial permeability for $3 \mathrm{kDa}$ fluorescein isothiocyanate-dextran and CXCL1induced basal-to-apical migration of neutrophils. Thus, Sumagin et al. [70"] suggest a detrimental feedback loop in the course of ulcerative colitis and Crohn's disease through engagement of ICAM-1 on the apical, that is, luminal face of the intestinal epithelial cells, which in turn increases neutrophil infiltration. 
Release of elastolytic proteases by neutrophils has a major impact on the course of inflammatory diseases in the lung (reviewed in [71]). Aggarwal et al. [72"] demonstrated increased expression of epithelial ICAM-1 and enhanced secretion of the neutrophil-attractant CXCL5 by epithelial cells, coinciding with increased trans-epithelial permeability and elevated numbers of alveolar neutrophils in cigarette smoke-induced emphysema in a mouse model [72"]. Mechanistically, the authors revealed that in the absence of the water channel protein AQP5 in AQP5 knockout mice, the increased epithelial ICAM-1 and CXCL5 expression was reversed. However, a direct link between neutrophil migration to alveolar spaces and epithelial ICAM-1 remains suggestive.

Zhao et al. [73] addressed the role of ICAM-1 for sepsis-induced lung injury and organ homing of neutrophils in a murine cecal ligation and puncture model [73]. ICAM-1 blockade via intravenous antibody injection strongly reduced bacterial burden and increased neutrophil counts in the blood and peritoneal lavage fluid while homing of the neutrophils to the lung, spleen or thymus was reduced and the organ immune status was improved. However, this study does not differentiate between neutrophil extravasation across the vascular endothelium or trans-epithelial migration. Nevertheless, inhibition of neutrophil homing to the organs via ICAM-1 blockade improved the outcome of sepsis.

\section{CONCLUSION}

Taking together, the important role of ICAM-1 and ICAM-2 acting in concert for trafficking of neutrophils to the site of inflammation is validated in a multitude of studies. However, clinical targeting of ICAM-1 and ICAM-2 to reduce neutrophil infiltration remains difficult due to additional roles of ICAM-1 in T-cell activation and ICAM-2 in angiogenesis. IVM [74], combined with sophisticated transgenic mouse models and in-vitro live cell imaging techniques, is under revolutionary development and will provide further new insights into the individual roles of ICAM-1 or ICAM-2 at distinct steps of neutrophil extravasation in the future.

\section{Acknowledgements}

We are grateful to Britta Engelhardt for critical reading of the manuscript.

\section{Financial support and sponsorship}

R.L. thankfully acknowledges financial support by the Foundation for Clinical-Experimental Cancer Research, Bern, Switzerland, and the Multiple Sclerosis Society, Zürich, Switzerland.

\section{Conflicts of interest}

There are no conflicts of interest.

\section{REFERENCES AND RECOMMENDED READING}

Papers of particular interest, published within the annual period of review, have been highlighted as:

- of special interest

-1 of outstanding interest

1. Summers $C$, Rankin $S M$, Condliffe $A M$, et al. Neutrophil kinetics in health and disease. Trends Immunol 2010; 31:318-324.

2. Borregaard N. Neutrophils, from marrow to microbes. Immunity 2010; 33:657-670.

3. Ramos TN, Bullard DC, Barnum SR. ICAM-1: isoforms and phenotypes.

- J Immunol 2014; 192:4469-4474

Review on ICAM-1 isoforms and the three different ICAM-1 knockout mouse lines.

4. $\mathrm{Xu} \mathrm{H}$, Tong IL, De Fougerolles $A R$, et al. Isolation, characterization, and expression of mouse ICAM-2 complementary and genomic DNA. J Immunol $1992 ; 149: 2650-2655$.

5. Sundd P, Gutierrez E, Koltsova EK, et al. 'Slings' enable neutrophil rolling at high shear. Nature 2012; 488:399-403.

6. Woodfin A, Voisin MB, Beyrau M, et al. The junctional adhesion molecule JAM-C regulates polarized transendothelial migration of neutrophils in vivo. Nat Immunol 2011; 12:761-769.

7. de Fougerolles AR, Stacker SA, Schwarting R, et al. Characterization of ICAM-2 and evidence for a third counter-receptor for LFA-1. J Exp Med 1991; 174:253-267.

8. Xiao L, Liu Y, Wang N. New paradigms in inflammatory signaling in vascular endothelial cells. Am J Physiol Heart Circ Physiol 2014; 306:H317-H325.

9. Halai $\mathrm{K}$, Whiteford J, Ma B, et al. ICAM-2 facilitates luminal interactions

n. between neutrophils and endothelial cells in vivo. J Cell Sci 2014; 127:620629.

Endothelial ICAM-2 fulfills a significant role for neutrophil crawling on the vascular endothelium and endothelial ICAM-2 is required for the initiation of paracellular diapedesis.

10. $\mathrm{Xu} \mathrm{H}$, Gonzalo JA, St Pierre $Y$, et al. Leukocytosis and resistance to septic shock in intercellular adhesion molecule 1-deficient mice. J Exp Med 1994; 180:95-109.

11. Sligh JE Jr, Ballantyne CM, Rich SS, et al. Inflammatory and immune responses are impaired in mice deficient in intercellular adhesion molecule 1. Proc Natl Acad Sci U S A 1993; 90:8529-8533.

12. Bullard $D C, H u X$, Schoeb $T R$, et al. Intercellular adhesion molecule-1 expression is required on multiple cell types for the development of experimental autoimmune encephalomyelitis. J Immunol 2007; 178:851-857.

13. Gerwin N, Gonzalo JA, Lloyd C, et al. Prolonged eosinophil accumulation in allergic lung interstitium of ICAM-2 deficient mice results in extended hyperresponsiveness. Immunity 1999; 10:9-19.

14. Gorina R, Lyck R, Vestweber $D$, et al. Beta2 integrin-mediated crawling on

a. endothelial ICAM-1 and ICAM-2 is a prerequisite for transcellular neutrophil diapedesis across the inflamed blood-brain barrier. J Immunol 2014; 192:324-337.

A sophisticated analysis on the individual roles of endothelial ICAM-1 and ICAM-2 and neutrophil LFA-1 and Mac-1 for the dynamic postarrest behaviour of neutrophils on an in-vitro BBB model.

15. Li R, Nortamo P, Valmu L, et al. A peptide from ICAM-2 binds to the leukocyte integrin CD11a/CD18 and inhibits endothelial cell adhesion. J Biol Chem 1993; 268:17513-17518.

16. Staunton DE, Dustin ML, Springer TA. Functional cloning of ICAM-2, a cell adhesion ligand for LFA-1 homologous to ICAM-1. Nature 1989; 339:61-64.

17. Xie J, Li R, Kotovuori $P$, et al. Intercellular adhesion molecule-2 (CD102) binds to the leukocyte integrin CD11b/CD18 through the A domain. J Immunol 1995; 155:3619-3628.

18. Jones $\mathrm{DH}$, Anderson $\mathrm{DC}$, Burr $\mathrm{BL}$, et al. Quantitation of intracellular Mac-1 (CD11b/CD18) pools in human neutrophils. J Leukoc Biol 1988; 44:535544.

19. Rose $\mathrm{DM}$, Alon $\mathrm{R}$, Ginsberg $\mathrm{MH}$. Integrin modulation and signaling in leukocyte adhesion and migration. Immunol Rev 2007; 218:126-134.

20. Luo BH, Carman CV, Springer TA. Structural basis of integrin regulation and signaling. Annu Rev Immunol 2007; 25:619-647.

21. Frommhold $D$, Kamphues $A$, Hepper $I$, et al. RAGE and ICAM-1 cooperate in mediating leukocyte recruitment during acute inflammation in vivo. Blood 2010; 116:841-849.

22. Arcangeli $M L$, Frontera $V$, Aurrand-Lions M. Function of junctional adhesion molecules (JAMs) in leukocyte migration and homeostasis. Arch Immunol Ther Exp (Warsz) 2013; 61:15-23.

23. Kolaczkowska $E$, Kubes $P$. Neutrophil recruitment and function in health and inflammation. Nat Rev Immunol 2013; 13:159-175.

Review on the lifecycle of neutrophils, their recruitment to infected or injured tissues and antimicrobial defence machinery. 
24. Schmidt S, Moser M, Sperandio M. The molecular basis of leukocyte - recruitment and its deficiencies. Mol Immunol 2013; 55:49-58.

Review on the three types of LAD.

25. Zarbock A, Ley K, McEver RP, et al. Leukocyte ligands for endothelial selectins: specialized glycoconjugates that mediate rolling and signaling under flow. Blood 2011; 118:6743-6751.

26. Lawrence MB, Kansas GS, Kunkel EJ, et al. Threshold levels of fluid shear promote leukocyte adhesion through selectins (CD62L,P,E). J Cell Biol 1997; 136:717-727.

27. Marshall BT, Long M, Piper JW, et al. Direct observation of catch bonds involving cell-adhesion molecules. Nature 2003; 423:190-193.

28. Sundd $P$, Pospieszalska MK, Ley $K$. Neutrophil rolling at high shear: flattening, catch bond behavior, tethers and slings. Mol Immunol 2013; 55:59-69.

Review on the molecular mechanism of initial contact formation between the neutrophil and the VE cells.

29. Moretti FA, Moser M, Lyck $R$, et al. Kindlin-3 regulates integrin activation and

- adhesion reinforcement of effector T cells. Proc Natl Acad Sci U S A 2013; 110:17005-17010.

Kindlin-3 is required for integrin activation in effector $T$ cells.

30. Futosi K, Fodor S, Mocsai A. Neutrophil cell surface receptors and their intracellular signal transduction pathways. Int Immunopharmacol 2013; $17: 638-650$

31. Voisin MB, Nourshargh S. Neutrophil transmigration: emergence of an adhesive cascade within venular walls. J Innate Immun 2013; 5:336347.

32. Sullivan DP, Muller WA. Neutrophil and monocyte recruitment by PECAM, CD99, and other molecules via the LBRC. Semin Immunopathol 2014; 36:193-209.

33. Giannotta M, Trani M, Dejana E. VE-cadherin and endothelial adherens junctions: active guardians of vascular integrity. Dev Cell 2013; 26:441 -454.

Review on the master regulator role of VE-cadherin for maturation of endothelial cell junctions.

34. Schulte D, Kuppers V, Dartsch N, et al. Stabilizing the VE-cadherin-catenin complex blocks leukocyte extravasation and vascular permeability. EMBO J 2011; 30:4157-4170.

35. Muller WA. Getting leukocytes to the site of inflammation. Vet Pathol 2013; 50:7-22.

36. Sage PT, Carman CV. Settings and mechanisms for trans-cellular diapedesis. Front Biosci 2009; 14:5066-5083.

37. Yang L, Froio RM, Sciuto TE, et al. ICAM-1 regulates neutrophil adhesion and transcellular migration of TNF-alpha-activated vascular endothelium under flow. Blood 2005; 106:584-592.

38. Carpen $O$, Pallai $P$, Staunton $D E$, et al. Association of intercellular adhesion molecule-1 (ICAM-1) with actin-containing cytoskeleton and alpha-actinin. J Cell Biol 1992; 118:1223-1234.

39. Kanters E, van Rijssel J, Hensbergen PJ, et al. Filamin B mediates ICAM-1driven leukocyte transendothelial migration. J Biol Chem 2008; 283:3183031839.

40. Yang L, Kowalski JR, Yacono $\mathrm{P}$, et al. Endothelial cell cortactin coordinates intercellular adhesion molecule-1 clustering and actin cytoskeleton remodeling during polymorphonuclear leukocyte adhesion and transmigration. J Immunol 2006; 177:6440-6449.

41. Etienne-Manneville $S$, Manneville JB, Adamson $P$, et al. ICAM-1-coupled cytoskeletal rearrangements and transendothelial lymphocyte migration involve intracellular calcium signaling in brain endothelial cell lines. J Immunol 2000; 165:3375-3383.

42. Heemskerk N, van Rijssel J, van Buul JD. Rho-GTPase signaling in leukocyte

- extravasation: an endothelial point of view. Cell Adh Migr 2014; 8:67-75.

Review on ICAM-1 and ICAM-2 intracellular signalling into the endothelial cells.

43. Schaefer A, Te Riet J, Ritz K, et al. Actin-binding proteins differentially regulate

- endothelial cell stiffness, ICAM-1 function and neutrophil transmigration. J Cell Sci 2014; 127:4470-4482.

ICAM-1 recruitment of the adaptor protein $\alpha$-Actinin- 4 regulates endothelial cell stiffness. Endothelial cells in atherosclerotic plaques are enriched in $\alpha$-Actinin- 4 .

44. Huynh J, Nishimura N, Rana K, et al. Age-related intimal stiffening enhances endothelial permeability and leukocyte transmigration. Sci Transl Med 2011; 3:; 112 ra122.

45. Heiska L, Kantor C, Parr T, et al. Binding of the cytoplasmic domain of intercellular adhesion molecule-2 (ICAM-2) to a-Actinin. J Biol Chem 1996; 42:26214-26219.

46. Heiska L, Alfthan $\mathrm{K}$, Gronholm M, et al. Association of ezrin with intercellular adhesion molecule-1 and -2 (ICAM-1 and ICAM-2). Regulation by phosphatidylinositol 4, 5-bisphosphate. J Biol Chem 1998; 273:21893-21900.

47. Yonemura S, Hirao M, Doi Y, et al. Ezrin/radixin/moesin (ERM) proteins bind to a positively charged amino acid cluster in the juxta-membrane cytoplasmic domain of CD44, CD43, and ICAM-2. J Cell Biol 1998; 140:885-895.

48. Amsellem V, Dryden NH, Martinelli R, et al. ICAM-2 regulates vascular

- permeability and $\mathrm{N}$-cadherin localization through ezrin-radixin-moesin (ERM) proteins and Rac-1 signalling. Cell Commun Signal 2014; 12:12.

Endothelial ICAM-2 is involved in maturation of endothelial cell junctions via recruitment of ERM proteins to its cytoplasmic domain and activation of Rac-1.

49. Phillipson $M$, Heit $B$, Colarusso $P$, et al. Intraluminal crawling of neutrophils to emigration sites: a molecularly distinct process from adhesion in the recruitment cascade. J Exp Med 2006; 203:2569-2575.
50. Hepper I, Schymeinsky J, Weckbach LT, et al. The mammalian actin-binding protein 1 is critical for spreading and intraluminal crawling of neutrophils under flow conditions. J Immunol 2012; 188:4590-4601.

51. Ransohoff RM, Engelhardt $B$. The anatomical and cellular basis of immune surveillance in the central nervous system. Nat Rev Immunol 2012; 12:623635.

52. Lyck R, Ruderisch N, Moll AG, et al. Culture-induced changes in blood-brain barrier transcriptome: implications for amino-acid transporters in vivo. J Cereb Blood Flow Metab 2009; 29:1491-1502.

53. Steiner $\mathrm{O}$, Coisne $\mathrm{C}$, Engelhardt $\mathrm{B}$, et al. Comparison of immortalized bEnd5 and primary mouse brain microvascular endothelial cells as in vitro bloodbrain barrier models for the study of T cell extravasation. J Cereb Blood Flow Metab 2011; 31:315-327.

54. Phillipson $M$, Heit $B$, Parsons $S A$, et al. Vav1 is essential for mechanotactic crawling and migration of neutrophils out of the inflamed microvasculature. J Immunol 2009; 182:6870-6878.

55. Valignat MP, Theodoly $O$, Gucciardi $A$, et al. $T$ lymphocytes orient against the direction of fluid flow during LFA-1-mediated migration. Biophys J 2013; 104:322-331.

56. Wedmore CV, Williams TJ. Control of vascular permeability by polymorphonuclear leukocytes in inflammation. Nature 1981; 289:646-650.

57. Arfors $\mathrm{KE}$, Lundberg $\mathrm{C}$, Lindbom $\mathrm{L}$, et al. A monoclonal antibody to the membrane glycoprotein complex CD18 inhibits polymorphonuclear leukocyte accumulation and plasma leakage in vivo. Blood 1987; 69:338-340.

58. Finsterbusch $M$, Voisin MB, Beyrau $M$, et al. Neutrophils recruited by chemoattractants in vivo induce microvascular plasma protein leakage through secretion of TNF. J Exp Med 2014; 211:1307-1314.

Neutrophils adherent via $\beta 2$-integrins on endothelial ICAM-1 or ICAM- 2 respond to C5a or CXCL1 with the release of TNF- $\alpha$.

59. Armulik A, Genove G, Betsholtz C. Pericytes: developmental, physiological, and pathological perspectives, problems, and promises. Dev Cell 2011; 21:193-215.

60. Stark K, Eckart A, Haidari S, et al. Capillary and arteriolar pericytes attract

- innate leukocytes exiting through venules and 'instruct' them with patternrecognition and motility programs. Nat Immunol 2013; 14:41-51.

Pericytes have different phenotypes. Only NG2 positive pericytes express ICAM-1 constitutively and attract neutrophils via secretion of MIF.

61. Ayres-Sander $\mathrm{CE}$, Lauridsen $\mathrm{H}$, Maier $\mathrm{CL}$, et al. Transendothelial migration enables subsequent transmigration of neutrophils through underlying pericytes. PLoS One 2013; 8:e60025.

Neutrophil migration across a pericyte layer is improved after transendothelial migration.

62. Proebstl D, Voisin MB, Woodfin A, et al. Pericytes support neutrophil subendothelial cell crawling and breaching of venular walls in vivo. J Exp Med 2012; 209:1219-1234.

63. Zen K, Parkos CA. Leukocyte-epithelial interactions. Curr Opin Cell Biol 2003; 15:557-564.

64. Parkos CA, Colgan SP, Diamond MS, et al. Expression and polarization of intercellular adhesion molecule-1 on human intestinal epithelia: consequences for CD11b/CD18-mediated interactions with neutrophils. Molec Med 1996; 2:489-505.

65. Parkos CA, Colgan SP, Liang TW, et al. CD47 mediates postadhesive events required for neutrophil migration across polarized intestinal epithelia. J Cell Biol 1996; 132:437-450.

66. Wewer $\mathrm{C}$, Seibt A, Wolburg $\mathrm{H}$, et al. Transcellular migration of neutrophil granulocytes through the blood-cerebrospinal fluid barrier after infection with Streptococcus suis. J Neuroinflamm 2011; 8:51.

67. Vainer $\mathrm{B}$, Nielsen $\mathrm{OH}$, Horn $\mathrm{T}$. Comparative studies of the colonic in situ expression of intercellular adhesion molecules (ICAM-1, -2, and -3), beta2 integrins (LFA-1, Mac-1, and p150,95), and PECAM-1 in ulcerative colitis and Crohn's disease. Am J Surg Pathol 2000; 24:1115-1124.

68. Steffen BJ, Breier G, Butcher EC, et al. ICAM-1, VCAM-1, and MAdCAM-1 are expressed on choroid plexus epithelium but not endothelium and mediate binding of lymphocytes in vitro. Am J Pathol 1996; 148:1819-1838.

69. Wolburg K, Gerhardt H, Schulz M, et al. Ultrastructural localization of adhesion molecules in the healthy and inflamed choroid plexus of the mouse. Cell Tissue Res 1999; 296:259-269.

70. Sumagin R, Robin AZ, Nusrat $A$, et al. Transmigrated neutrophils in the

intestinal lumen engage ICAM-1 to regulate the epithelial barrier and neutrophil recruitment. Mucosal Immunol 2014; 7:905-915.

Neutrophils engage ICAM-1 on the apical face of epithelial cells and facilitate basal-to-apical migration of neutrophils that follow.

71. Sandhaus RA, Turino G. Neutrophil elastase-mediated lung disease. COPD 2013; 10 (Suppl 1):60-63.

72. Aggarwal NR, Chau E, Garibaldi BT, et al. Aquaporin 5 regulates cigarette

- smoke induced emphysema by modulating barrier and immune properties of the epithelium. Tissue Barriers 2013; $1: \mathrm{e} 25248$.

Demonstrates a link between aquaporin 5, alveolar neutrophil infiltration and epithelial ICAM-1.

73. Zhao YJ, Yi WJ, Wan XJ, et al. Blockade of ICAM-1 improves the outcome of polymicrobial sepsis via modulating neutrophil migration and reversing immunosuppression. Mediators Inflamm 2014; 2014:195290.

74. Zarbock A, Ley K. New insights into leukocyte recruitment by intravital microscopy. Curr Top Microbiol Immunol 2009; 334:129-152. 